\title{
ASSESSMENT OF POSTURAL INSTABILITY IN PATIENTS WITH A NEUROLOGICAL DISORDER USING A TRI-AXIAL ACCELEROMETER
}

\author{
Lenka Hanakova $^{a, *}$, Vladimir Socha $^{a}$, Jakub Schlenker $^{a}$, \\ OndREJ CAKRT ${ }^{a, b}$, PATRIK KUTILEK ${ }^{a}$ \\ ${ }^{a}$ Faculty of Biomedical Engineering, Czech Technical University in Prague, nam. Sitna 3105, Kladno, Czech \\ Republic \\ ${ }^{b}$ 2nd Faculty of Medicine, University Hospital Motol, Charles University in Prague, V Uvalu 84, Prague, Czech \\ Republic \\ * corresponding author: lenka.hanakova@fbmi.cvut.cz
}

\begin{abstract}
Current techniques for quantifying human postural stability during quiet standing have several limitations. The main problem is that only two movement variables are evaluated, though a better description of complex three-dimensional (3-D) movements can be provided with the use of three variables. A single tri-axial accelerometer placed on the trunk was used to measure 3-D data. We are able to evaluate 3-D movements using a method based on the volume of confidence ellipsoid (VE) of the set of points obtained by plotting three accelerations against each other. Our method was used to identify and evaluate pathological balance control. In this study, measurements were made of patients with progressive cerebellar ataxia, and also control measurements of healthy subjects, and a statistical analysis was performed. The results show that the VEs of the neurological disorder patients are significantly larger than the VEs of the healthy subjects. It can be seen that the quantitative method based on VE is very sensitive for identifying changes in stability, and that it is able to distinguish between neurological disorder patients and healthy subjects.
\end{abstract}

KEYWORDS: trunk acceleration, gyro-accelerometer, postural stability, confidence ellipsoid volume, cerebellar disease.

\section{INTRODUCTION}

Neurological disorders usually have a negative effect on trunk posture [1]3. Patients with neurological disorders often show instability during quiet stance tasks. In recent years, tri-axial inertial measurement units (IMU) for acceleration and orientation measurements have been replacing force platforms for high-accuracy 3 -D measurements of body movements for studying the centre of pressure (CoP) excursions [4] of patients with neurological disorders. The sensing units can be used to measure the three Euler angles and the three accelerations of a segment. In the past, sensing units were placed on top of the head, spinous processes of $\mathrm{T} 1$ and/or $\mathrm{S} 1$ in order to measure the motion of the head, the trunk and the pelvis, respectively. Although IMU systems can measure the three angles and the three accelerations, techniques for quantifying segment movements using only one or two measured quantities were introduced into clinical practice [4 8 ]. Measurements of trunk accelerations during stance can identify impaired balance control in individuals with neurological disorders [5, 9]. For posture monitoring and training, a non-commercial IMU system has also been developed as a diagnostic tool for trunk movements [10, 11. In addition, the cheap accelerometers and gyroscopes used in modern cell phones have been tested for posture monitoring [6]. An assessment of trunk movements using IMU may yield clearer insights into balance deficits, and may provide a considerably better diagnostic tool than the more traditional measures mentioned above. Although measurements of trunk movements can provide aid in diagnostics and rehabilitation, measurements of trunk movements using IMU have not previously been implemented in the study of numerous postural balance problems of patients with specific types of diseases. The force (posturography) platform has until now been the main tool for studying, e.g., the body movement of patients suffering from cerebellar diseases [12]. Traditional, more complex methods for processing the measured data and for assessing postural instability, using at least two measured variables, are based on the 2-D convex hull [13-15], the 2-D confidence ellipse [16-18, or the length of the trajectory obtained by plotting two variables against each other [19, 20]. These methods are usually used to evaluate the data from the force platform [12, 21]. However, these traditional ways of quantifying postural stability have some limitations. The major limitation is that the methods are based on evaluating only two variables, one in each of the two planes/axes of the human body. This can lead to a loss of important information about physical activity, specifically the third physical quantity of 3-D movements. Although tri-axial IMUs have been used 
for quantifying postural instability in clinical practice, the methods for evaluating the measured data usually use only two variables out of the three measured variables.

This paper aims to validate and apply a method for identifying the pathological balance control of patients with a neurological disorder on the basis of measurements of accelerations by IMU, and the volume of confidence ellipsoid of the set of points obtained by plotting the three accelerations, measured during quiet stance, against each other. The technique relies on a well-known principle that is used for evaluating the 2-D data set [16-18]. The reason for applying this method is that a study of three-dimensional movement with one variable characterizing the change in all three accelerations may find new uses for the study of postural stability in clinical practice using a lowcost tri-axial IMU. Moreover, these accelerations are directly related to changes in position, which means that we could make a direct evaluation of the accelerations. This method is used for diagnosing cerebellar disease manifested by tremors or by swaying, since it is primarily designed for use in neurology to discover relationships between neurological disorders and postural trunk movements in 3-D space.

\section{Methods AND MATERIALS}

\subsection{PARTICIPANTS}

Ten volunteer patients (aged 52.2 (SD 11.7) years) with degenerative cerebellar ataxia [22] and eleven healthy subjects (aged 26.0 (SD 6.4) years) participated in the study. A comparison of groups of the same age is not necessary in this case, since studies have shown that the body sway parameters of healthy subjects within the age range between 20 and 60 years vary only slightly $[23,24$. Aoki et al. 23] found that there were insignificant differences in 10-60-year-old subjects in COP sway parameters: circumference, rectangular area, left-right width, and front-back width in both cases (eyes open/eyes closed), i.e., no significant age-related differences were found in all the COP sway parameters (i.e., Romberg quotients). It has been shown that significantly degraded stability begins after the age of 60 years. In addition, a detailed analysis of an age-related increase in the CoP parameters by polynomial-type regression showed that a gradual increase in body sway, characterized by increased CoP oscillations, begins around the age of 60 years [24].

The patients were recruited from the Motol Faculty Hospital, Prague, Czech Republic. A board-certified neurologist had previously screened and diagnosed progressive cerebellar disease. The diagnostic evaluation included a detailed disease history, a neurological examination, routine laboratory blood and urine tests, and a brain MRI. The patients were measured in the initial phase of the clinic's two-week rehabilitation program. Healthy subjects were recruited from

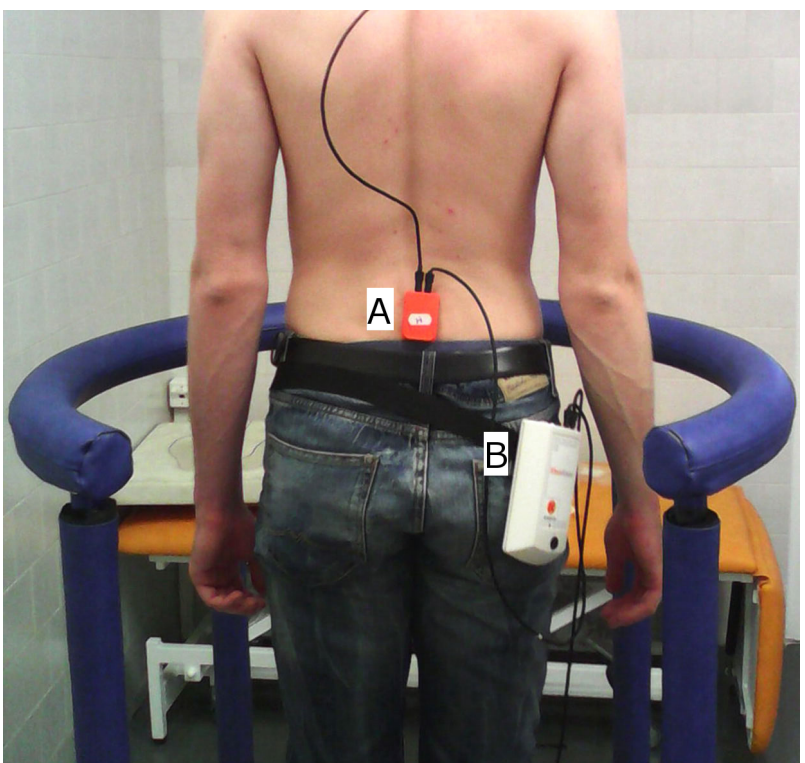

Figure 1. The Xsens system with one tri-axial IMU (A) and a central unit (B) used to measure angles and accelerations of the trunk.

students/volunteers at Charles University in Prague. For the healthy subjects, the diagnostic evaluation included a detailed disease history, a neurological examination and routine laboratory testing. The study was performed in accordance with the Helsinki Declaration. The study protocol was approved by the local Ethics Committee and the Motol University Hospital, and informed consent was obtained from all subjects. The subjects were chosen for the measurements randomly, and on different days.

\subsection{TEST PROCEDURE AND MEASUREMENT EQUIPMENT}

The Xsens system, composed of the Xbus Master (central unit), a lightweight (330g) portable device using MTx units (tri-axial IMUs) for orientation and acceleration measurement of body segments, was used for measuring trunk movements (see Fig. 1). The MTx unit is an accurate IMU providing drift-free 3-D orientation and 3-D acceleration. The MTx unit was calibrated before each measurement, and was set up in such a way that one axis of the coordinate system was parallel to the anterior-posterior axis, i.e., the axis of symmetry of the fixed stationary platform on which the participants stood, and the other two axes were perpendicular to the anterior-posterior axis (i.e., the axis of symmetry of the platform) with respect to the direction of the Earth's gravitational field, i.e., the anterior-posterior axis was co-linear with the direction of gravity. After calibration, one MTx unit was placed on the patient's trunk, according to [ $[$, 13, 25], at the level of the lower back (lumbar 2-3), see Fig. 1 .

The data, i.e., the three angles (Euler angles: roll, yaw and pitch) 26-28] and the three accelerations (in the accelerometer coordinate system) [29] of the trunk, were measured by the IMU while the patients 
(Pts) and the healthy subjects (control group - CG) were standing quietly standing on a fixed stationary platform. In brief, trunk sway was measured during quiet stance on a firm surface ( $\mathrm{FiS}$ ) and on a soft foam surface (FoS) with eyes open (EO) and with eyes closed (EC) 30. The tasks included standing on each legg for at least 60 seconds 31. The measurements usually lasted for a few seconds longer, and the initial data were cut off, so that all datasets have a record length of 60 seconds. The subject's bare feet were positioned next to each other, splayed at an angle of $30^{\circ}$, with the arms always in hanging position. The three angles, the three accelerations and the task duration were recorded with a sample frequency of $100 \mathrm{~Hz}$. There was no need to normalize the data measured during quiet stance because the standard ranges of angles and accelerations are the same for all three planes of the body and for all adult subjects, since the IMU was placed on the same anatomical point.

\subsection{DATA ANALYSIS}

The roll $(\Phi)$, yaw $(\Psi)$ and pitch $(\Theta)$ angles and the three orthogonal accelerations $\left(a_{S x}, a_{S y}, a_{S z}\right)$ in the accelerometer coordinate system [29] were measured by one MTx unit. The conventions of the Euler angles (roll, yaw and pitch) are described in [27, 28, 32]. The three accelerations measured by the accelerometer of the IMU (i.e., the MTx unit) are described in detail in 33,34 . These angles and accelerations are used to calculate the accelerations in the global reference system and then in the anatomical coordinate frame. The calculation is based on the rotational matrices. The first rotation matrix $R_{G S}$ can be interpreted in terms of the Euler angles [35]:

$$
R_{G S}=R_{\Psi}^{Z} \cdot R_{\Theta}^{Y} \cdot R_{\Phi}^{X}
$$

where

$$
\begin{aligned}
& R_{\Psi}^{Z}=\left[\begin{array}{ccc}
\cos \Psi & -\sin \Psi & 0 \\
\sin \Psi & \cos \Psi & 0 \\
0 & 0 & 1
\end{array}\right], \\
& R_{\Theta}^{Y}=\left[\begin{array}{ccc}
\cos \Theta & 0 & \sin \Theta \\
0 & 1 & 0 \\
-\sin \Theta & 0 & \cos \Theta
\end{array}\right], \\
& R_{\Phi}^{X}=\left[\begin{array}{ccc}
1 & 0 & 0 \\
0 & \cos \Phi & -\sin \Phi \\
0 & \sin \Phi & \cos \Phi
\end{array}\right] .
\end{aligned}
$$

Matrix $R_{G S}$ rotates an acceleration vector $\overrightarrow{a_{S}}=$ $\left(a_{S x}, a_{S y}, a_{S z}\right)^{T}$ in the sensor co-ordinate system $(S)$ to the global reference system $(G)$ :

$$
\overrightarrow{a_{G}}=R_{G S} \cdot \overrightarrow{a_{S}}
$$

The acceleration vector $\overrightarrow{a_{G}}=\left(a_{G x}, a_{G y}, a_{G z}\right)^{T}$ in the global reference system is then rotated by second

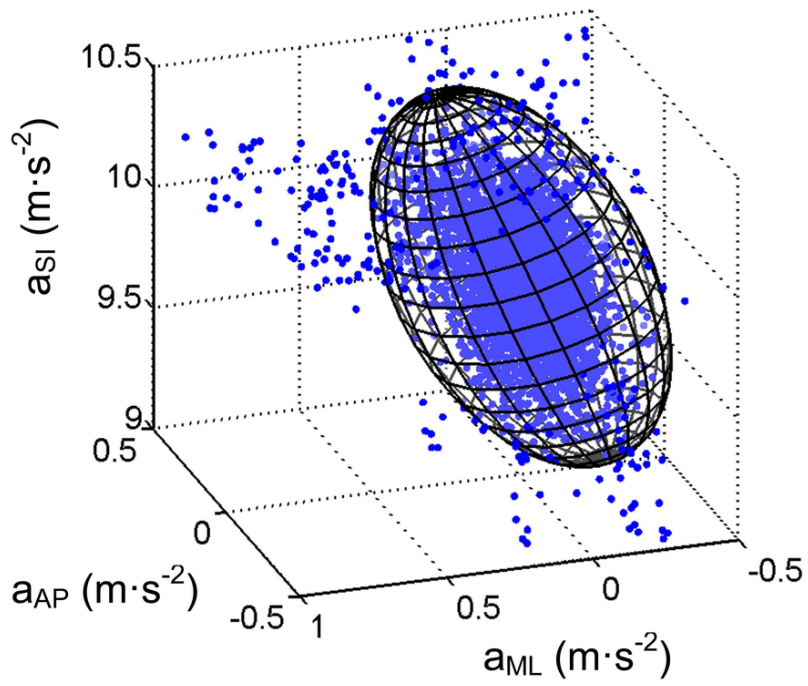

Figure 2. Example of the confidence ellipsoid obtained by plotting superior-inferior (SI), medio-lateral $(\mathrm{ML})$ and anterior-posterior (AP) accelerations against each other.

rotation matrix $R_{A G}$ to the anatomical coordinate frame $(A)$ [36]:

$$
\overrightarrow{a_{A}}=R_{A G} \cdot \vec{G}
$$

where

$$
R_{A G}=R_{\Psi 0}^{Z}=\left[\begin{array}{ccc}
\cos \Psi_{0} & -\sin \Psi_{0} & 0 \\
\sin \Psi_{0} & \cos \Psi_{0} & 0 \\
0 & 0 & 1
\end{array}\right]
$$

The angle $\Psi_{0}$ is obtained during the calibration process of the MTx unit. The calculated acceleration vector $\overrightarrow{a_{A}}=\left(a_{A P}, a_{M L}, a_{S I}\right)^{T}$ in the anatomical coordinate frame represents the superior-inferior acceleration $\left(a_{S I}\right)$, the medio-lateral acceleration $\left(a_{M L}\right)$ and the anterior-posterior acceleration $\left(a_{A P}\right)$.

Using the accelerations derived above we are able to evaluate the $3-\mathrm{D}$ movements of the patients. The calculated acceleration vectors, or in other words, the time dependent data $\left(a_{S I}, a_{M L}, a_{A P}\right)$ obtained by the tri-axal accelerometer are plotted as a 3-D plot, i.e., a set of points is obtained by plotting the three accelerations against each other, see Fig. 2. The number of points is determined by the time of the measurements, i.e., the recorded length of the dataset $(60 \mathrm{~s})$ and the sample frequency $(100 \mathrm{~Hz})$.

The method for identifying pathological balance control is based on the mathematical tools for static posturography, which are used to analyze the body sway of unsteady patients $16-18$. We can model the distribution of the measured data by a $2-\mathrm{D}$ ellipse or a 3-D ellipsoid. The confidence ellipse area has already been used in clinical practice to study postural balance problems, but the concept of the confidence ellipsoid volume has not been used before in clinical practice for studying the postural balance problems by three accelerations. We have used a method based 


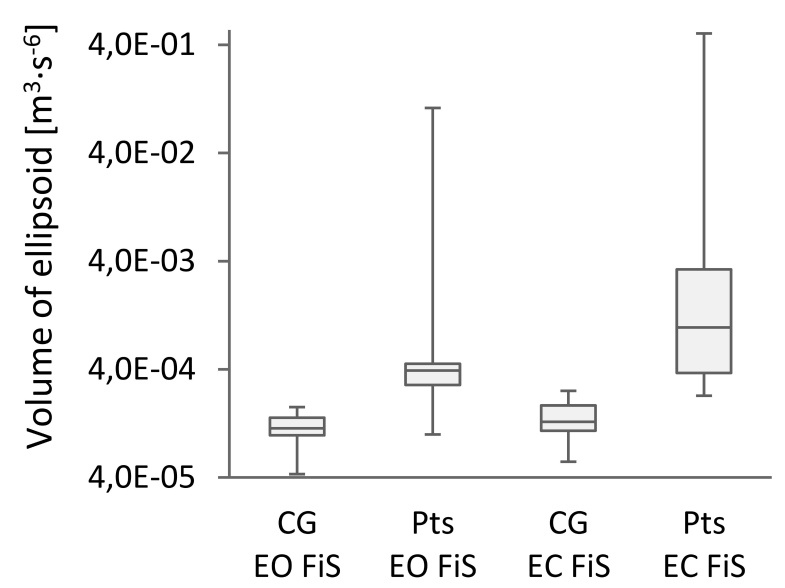

Figure 3. Comparison of the volume of the ellipsoid of the control group (CG) and the patients (Pts) standing on a firm surface (FiS), with eyes open (EO) and with eyes closed (EC).

on a description of the distribution of the measured data (i.e., $a_{S I}, a_{M L}$ and $a_{A P}$ ) by a confidence ellipsoid. The confidence ellipsoid is determined using the Principal Component Analysis (PCA) [37. The volume of an ellipsoid is given by an ellipsoid matrix and the ellipsoid matrix is composed of entries from the covariance matrix [38, 39. The symmetric $3 \times 3$ covariance matrix is given by the equation [40, 41]:

$$
C=\left[\begin{array}{ccc}
\operatorname{Var}\left(a_{A P}\right) & \operatorname{Cov}\left(a_{A P}, a_{M L}\right) & \operatorname{Cov}\left(a_{A P}, a_{S I}\right) \\
\operatorname{Cov}\left(a_{M L}, a_{A P}\right) & \operatorname{Var}\left(a_{M L}\right) & \operatorname{Cov}\left(a_{M L}, a_{S I}\right) \\
\operatorname{Cov}\left(a_{S I}, a_{A P}\right) & \operatorname{Cov}\left(a_{S I}, a_{M L}\right) & \operatorname{Var}\left(a_{S I}\right)
\end{array}\right]
$$

The eigensystem of the covariance matrix constitutes a quadratic surface which is used for visualization. The confidence ellipsoid that makes the fewest assumptions about the shape of the underlying distribution from which the measured data can be drawn is a three-dimensional ellipsoid, Fig. 2 We use a 95\% confidence ellipsoid $(\mathrm{CE})$ to verify the applicability of a 3-D confidence ellipsoid [42 44. The 95\% confidence ellipsoid (with 0.95 probability [45, 46]) volume is the volume of an ellipsoid that is expected to bound $95 \%$ of the measured data, i.e., the set of points is obtained by plotting three accelerations against each other. The confidence ellipsoid volume is given by the equation:

$$
V=\frac{4}{3} \pi a b c,
$$

where $a=\sqrt{\chi^{2} \lambda_{1}}, b=\sqrt{\chi^{2} \lambda_{2}}, c=\sqrt{\chi^{2} \lambda_{3}}$ are semiaxes, $\lambda_{1,2,3}$ are the eigenvalues of matrix $C$, and $\chi^{2}=5.991$ as reported by Greenwalt, C. R. and Schultz [47. In our case, the physical unit of the volume is $\mathrm{m}^{3} \mathrm{~s}^{-6}$. Although the MTx unit also senses the gravitational acceleration it is not necessary to subtract the gravitational acceleration because the method for calculating VE uses only changes in the accelerations, and the gravitational acceleration is constant and perpendicular to the horizontal plane of the Earth's surface.

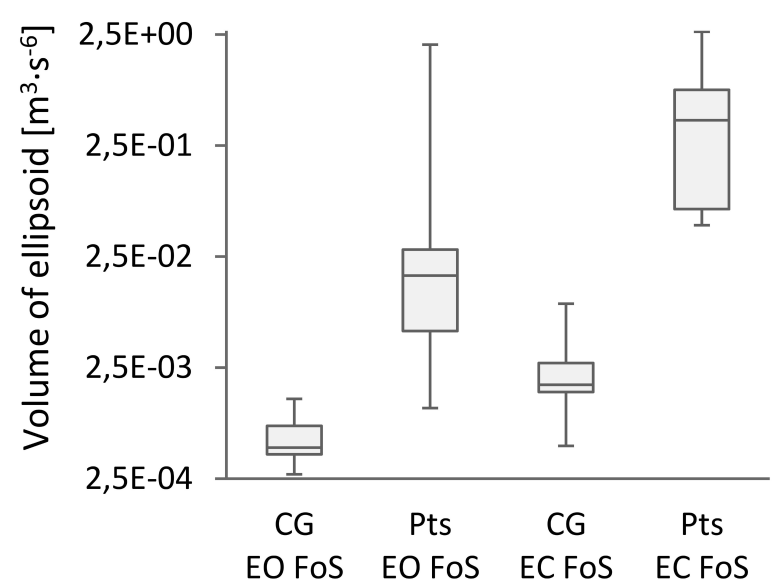

Figure 4. Comparison of the volume of the ellipsoid of the control group (CG) and the patients (Pts) standing on a foam surface (FoS) with eyes open (EO) and with eyes closed (EC).

All calculations were performed using customwritten MatLab programs with principal component analysis implemented in MatLab software (MatLab R2010b, Mathworks, Inc., Natick, MA, USA) to determine the symmetric $3 \times 3$ covariance matrix of the measured data and the confidence ellipsoid volume.

\subsection{Statistical ANALYSIS}

After calculating the VE of each patient and of each healthy subject standing on an FiS and on an FoS with EO and EC, the statistical analysis was performed using MatLab software. The Jarque-Bera test was used to test the normal distribution of the calculated VEs [48. The test returns a value of $h=1$ if it rejects the null hypothesis at the 0.05 significance level, and $h=0$ otherwise. The median (Mdn), minimum (Min), maximum (Max), the first quartile (Q1) and the third quartile (Q3) of the AV and TL are then used to compare the results for Pts and CG. In addition, the Wilcoxon signed rank test and the Wilcoxon rank sum test [49] were used to assess the significance of the differences between the results of the measurements. The significance level was set at $p<0.05$.

\section{Results}

The statistical data are used to illustrate the differences between the VEs of Pts with EO, Pts with EC, CG with EO and CG with EC (Tab. 1). The following plots (Fig. 3 and Fig. 4) display the Min, Max, Mdn, Q1, and Q3 for the VEs. Since some calculated values were not distributed normally, the Wilcoxon test was used to compare and analyze sets of calculated values.

The comparison of CG on FiS with EO and CG on FiS with EC $(p=0.18)$ revealed no differences. Differences were found when comparing Pts on FiS with EO and Pts on FiS with EC $(p=0.02)$, CG on FoS with EO and CG on FoS with EC $(p<0.01)$, and Pts on FoS with EO and Pts on FoS with EC 


\begin{tabular}{cccccc}
\hline & & CG EO & Pts EO & CG EC & Pts EC \\
\hline FiS & Min & $4.30 \cdot 10^{-5}$ & $1.00 \cdot 10^{-4}$ & $5.60 \cdot 10^{-5}$ & $2.28 \cdot 10^{-4}$ \\
\cline { 2 - 6 } & Max & $1.79 \cdot 10^{-4}$ & $1.04 \cdot 10^{-1}$ & $2.53 \cdot 10^{-4}$ & $5.10 \cdot 10^{-1}$ \\
\cline { 2 - 6 } & Mdn & $1.14 \cdot 10^{-4}$ & $3.90 \cdot 10^{-4}$ & $1.31 \cdot 10^{-4}$ & $9.76 \cdot 10^{-4}$ \\
\cline { 2 - 6 } & Q1 & $9.80 \cdot 10^{-5}$ & $2.86 \cdot 10^{-4}$ & $1.09 \cdot 10^{-4}$ & $3.70 \cdot 10^{-4}$ \\
\cline { 2 - 6 } FoS & Q3 & $1.43 \cdot 10^{-4}$ & $4.49 \cdot 10^{-4}$ & $1.85 \cdot 10^{-4}$ & $3.36 \cdot 10^{-3}$ \\
\cline { 2 - 6 } & Max & $2.74 \cdot 10^{-4}$ & $1.08 \cdot 10^{-3}$ & $4.94 \cdot 10^{-4}$ & $4.80 \cdot 10^{-2}$ \\
\cline { 2 - 6 } & Mdn & $4.76 \cdot 10^{-4}$ & $1.68 \cdot 10^{-2}$ & $1.76 \cdot 10^{-3}$ & $4.23 \cdot 10^{-1}$ \\
\cline { 2 - 6 } & Q1 & $4.15 \cdot 10^{-4}$ & $5.34 \cdot 10^{-3}$ & $1.51 \cdot 10^{-3}$ & $6.71 \cdot 10^{-2}$ \\
\cline { 2 - 6 } & Q3 & $7.48 \cdot 10^{-4}$ & $2.89 \cdot 10^{-2}$ & $2.76 \cdot 10^{-3}$ & $7.93 \cdot 10^{-1}$ \\
\hline
\end{tabular}

TABLE 1. Comparison of the volume of the ellipsoids of the control group and the patients (all values in $\mathrm{m}^{3} \mathrm{~s}^{-6}$ ).

$(p<0.01)$. For the subjects standing on FiS, the measured data of the CG or Pts with EO and EC show the same median or a slight increase in the median of the VEs after the eyes were closed (Fig. 3). For the subjects standing on FoS, the measured data for the CG or Pts with EO and EC show a significant increase in the median of the VEs when the eyes were closed (Fig. 4).

Differences were found when comparing CG on FiS with EO and Pts on FiS with EO $(p<0.01)$ show a significant difference, CG on FiS with EC and Pts on FiS with EC $(p<0.01), \mathrm{CG}$ on FoS with EO and Pts on FoS with EO $(p<0.01)$, and CG on FoS with EC and Pts on FoS with EC $(p<0.01)$. It can therefore be concluded, that there is significant difference between the data for CG and for Pts.

The method based on the VE provides significantly different results if the CG and Pts are measured when standing on both types of surface. The median of the VEs in Pts standing on FiS with EO is 3.4 times larger than the median of the VEs for CG standing on FiS with EO. The median of the VEs in Pts standing on FiS with EC is 7.5 times higher than the median of the VEs in CG standing on FiS with EC. The median of the VEs in Pts standing on FoS with EO is 35.3 times higher than the median of the VEs in CG standing on FoS with EO. The median of the VEs in Pts standing on FoS with EC is 240.3 times higher than the median of the VEs in CG standing on FoS with EC.

\section{Discussion}

A technique based on the volume of the confidence ellipsoid of the set of points obtained by plotting three accelerations against each other has been tested in clinical practice. The results for the method show that in all cases where the subjects stand on FiS or FoS the VEs in Pts with progressive cerebellar ataxia are significantly higher than the VEs in healthy subjects. However, the differences are much more pronounced when standing with EC on FoS. For patients or healthy subjects standing on FoS with EO and EC, the findings are consistent with findings obtained by traditional methods [13, 50, and show a significant difference between the postural stability of Pts and CG. These findings also justify the decision to ask the subjects stand on a foam surface with EC during the medical examination [51, 52].

For Pts standing on FoS with EC, the median of the $\mathrm{VE}$ is $4.23 \cdot 10^{-1} \mathrm{~m}^{3} \mathrm{~s}^{-6}$. For healthy subjects, the median of the $\mathrm{VE}$ is only $1.76 \cdot 10^{-3} \mathrm{~m}^{3} \mathrm{~s}^{-6}$. The results show that our method clearly identifies a deterioration in postural control, and large differences between the postural stability of Pts and CG. This difference is caused by a strong tremor or swaying of patients' trunks, which is one of the manifestations of cerebellar diseases [22, 50]. According to the method presented here, VE can yield insights into the postural stability and postural balance problems in patients with neurological disorders. This information is important in medical examinations and in rehabilitation medicine. The method based on VE can therefore be used as an additional method for determining and evaluating of postural instability. The primary advantage of the method is that the use of a cheap tri-axial IMU enables us to study the patient's trunk movements in all three planes/axes of the human body.

There are potential limitations to our study. The most important limitation is that the sample of subjects was small, and was probably not representative of the population as a whole. Although statistically significant results were observed, it would be helpful to see these findings proven in a larger study. However, a sample of ten Pts and eleven CG was sufficient to test the basic features of the techniques proposed for the study of the Pts with degenerative cerebellar disorder in this preliminary study. The proposed techniques should be seen as a contribution to the study of the postural stability of Pts with a neurological disorder, for which widely used method based on cheap tri-axial IMU is not adopted. 


\section{Conclusions}

We have carried out a study of postural instability in patients with a neurological disorder based on the volume of a confidence ellipsoid of the set of points obtained by plotting three accelerations against each other, and have shown that our technique is suitable for identifying postural balance problems. The technique enables us to study the distribution of the three measured variables (superior-inferior acceleration, medio-lateral acceleration and anterior-posterior acceleration) of body movements, and to overcome the greatest limitation of traditional methods, which rely only on two variables, one in each of the axes of the human body (medio-lateral and anterior-posterior). This method is used for studying the COP. Our new technique, based on the confidence ellipse volume, has never been used before for studying patients diagnosed with neurological disorders. The findings have shown that a single inexpensive tri-axial accelerometer placed on the patient's body segments can be a reliable tool for clinical measurements, and that our method more suitable than the force platforms that are currently widely used [4. Finally, it should be mentioned that our new technique can also be used for analyzing data obtained using the accelerometers installed in cell phones or in electronic watches. This can make it possible to use the methods proposed here in small health clinics or even in the patient's home.

\section{ACKNOWLEDGEMENTS}

This work was done in the Joint Department of the Faculty of Biomedical Engineering of the Czech Technical University in Prague and Charles University in Prague in the framework of research program VG20102015002 (2010-2015, MV0/VG), sponsored by the Ministry of the Interior of the Czech Republic, and project SGS15/107/OHK4/1T/17 of the CTU in Prague.

\section{REFERENCES}

[1] O. Cakrt et al. Exercise with visual feedback improves postural stability after vestibular schwannoma surgery. European Archives of Oto-Rhino-Laryngology 267(9):1355-1360, 2010. http://dx.doi.org/10.1007/s00405-010-1227-x, DOI:10.1007/s00405-010-1227-x.

[2] K. Bridgewater, M. Sharpe. Trunk muscle performance in early parkinson's disease. Physical Therapy 78(6):566-576, 1998.

[3] M. Latt et al. Acceleration patterns of the head and pelvis during gait in older people with parkinson's disease: a comparison of fallers and nonfallers. The Journals of Gerontology Series A: Biological Sciences and Medical Sciences 64A(6):700-706, 2009. http://dx.doi.org/10.1093/gerona/glp009 DOI:10.1093/gerona/glp009.

[4] M. Mancini et al. Isway: a sensitive, valid and reliable measure of postural control. J Neuroeng Rehabil 9(59):59, 2012. http://dx.doi.org/10.1186/1743-0003-9-59, DOI:10.1186/1743-0003-9-59
[5] J. Liu, X. Zhang, T. Lockhart. Fall risk assessments based on postural and dynamic stability using inertial measurement unit. Safety and Health at Work 3(3):192-198, 2012.

http://dx.doi.org/10.5491/shaw.2012.3.3.192 DOI:10.5491/shaw.2012.3.3.192

[6] B.-C. Lee et al. Cell phone based balance trainer. Journal of NeuroEngineering and Rehabilitation 9(1):10, 2012. http://dx.doi.org/10.1186/1743-0003-9-10, DOI:10.1186/1743-0003-9-10

[7] J. Allum et al. Trunk sway measures of postural stability during clinical balance tests: effects of a unilateral vestibular deficit. Gait \&S Posture

14(3):227-237, 2001. http:

//dx.doi.org/10.1016/s0966-6362(01)00132-1 DOI:10.1016/s0966-6362(01)00132-1.

[8] A. L. Adkin, B. R. Bloem, J. H. J. Allum. Trunk sway measurements during stance and gait tasks in parkinson's disease. Gait \& Posture 22(3):240-249, 2005. http: //dx.doi.org/10.1016/j.gaitpost.2004.09.009 DOI:10.1016/j.gaitpost.2004.09.009

[9] W. Maetzler et al. Impaired trunk stability in individuals at high risk for parkinson's disease. PLoS ONE 7(3):e32240, 2012. http://dx.doi.org/10.1371/journal.pone.0032240 DOI:10.1371/journal.pone.0032240

[10] E. Lou et al. A low power accelerometer used to improve posture. In Canadian Conference on Electrical and Computer Engineering 2001. Conference Proceedings (Cat. No.01TH8555). 2001. DOI:10.1109/ccece.2001.933657

[11] W. Wong, M. Wong. Trunk posture monitoring with inertial sensors. European Spine Journal 17(5):743-753, 2008.

http://dx.doi.org/10.1007/s00586-008-0586-0, DOI:10.1007/s00586-008-0586-0.

[12] S. Kammermeier et al. Disturbed vestibular-neck interaction in cerebellar disease. $J$ Neurol 260(3):794-804, 2012. http://dx.doi.org/10.1007/s00415-012-6707-z, DOI:10.1007/s00415-012-6707-z.

[13] B. Van de Warrenburg et al. Trunk sway in patients with spinocerebellar ataxia. Movement Disorders 20(8):1006-1013, 2005.

http://dx.doi.org/10.1002/mds.20486 DOI:10.1002/mds.20486

[14] E. de Hoon et al. Quantitative assessment of the stops walking while talking test in the elderly. Archives of Physical Medicine and Rehabilitation 84(6):838-842, 2003. http:

//dx.doi.org/10.1016/s0003-9993(02)04951-1 DOI:10.1016/s0003-9993(02)04951-1.

[15] C. Horlings et al. Identifying deficits in balance control following vestibular or proprioceptive loss using posturographic analysis of stance tasks. Clinical Neurophysiology 119(10):2338-2346, 2008. http://dx.doi.org/10.1016/j.clinph.2008.07.221 DOI:10.1016/j.clinph.2008.07.221.

[16] P. Odenrick, P. Sandstedt. Development of postural sway in the normal child. Human neurobiology 3(4):241-244, 1983. 
[17] T. Prieto, J. Myklebust, B. Myklebust. Postural steadiness and ankle joint compliance in the elderly. IEEE Eng Med Biol Mag 11(4):25-27, 1992. http://dx.doi.org/10.1109/51.256953 DOI:10.1109/51.256953

[18] L. Oliveira, D. Simpson, J. Nadal. Calculation of area of stabilometric signals using principal component analysis. Physiological Measurement 17(4):305-312, 1996. http://dx.doi.org/10.1088/0967-3334/17/4/008 DOI:10.1088/0967-3334/17/4/008

[19] J. Raymakers, M. Samson, H. Verhaar. The assessment of body sway and the choice of the stability parameter(s). Gait \& Posture 21(1):48-58, 2005. http: //dx.doi.org/10.1016/j.gaitpost.2003.11.006 DOI:10.1016/j.gaitpost.2003.11.006

[20] C. Horlings et al. Influence of virtual reality on postural stability during movements of quiet stance. Neuroscience Letters 451(3):227-231, 2009. http://dx.doi.org/10.1016/j.neulet.2008.12.057 DOI:10.1016/j.neulet.2008.12.057.

[21] M. Manto. Cerebellar, Disorders: A Practical Approach to Diagnosis and Management. Cambridge University Press, 2010.

[22] O. Cakrt et al. Balance rehabilitation therapy by tongue electrotactile biofeedback in patients with degenerative cerebellar disease. NeuroRehabilitation 31(4):429-434, 2012.

[23] H. Aoki. Evaluating the effects of open/closed eyes and age-related differences on center of foot pressure sway during stepping at a set tempo. Advances in Aging Research 1(3): 72-77, 2012.

http://dx.doi.org/10.4236/aar.2012.13009, DOI:10.4236/aar.2012.13009

[24] D. Abrahamova, F. Hlavacka. Age-related changes of human balance during quiet stance. Physiological Research 57(6):957, 2008.

[25] F. Ochi, K. Abe, S. Ishigami, et al. Trunk motion analysis in walking using gyro sensors. In Proceedings of the 19th Annual International Conference of the IEEE Engineering in Medicine and Biology Society.

'Magnificent Milestones and Emerging Opportunities in Medical Engineering' (Cat. No.97CH36136). 1997. DOI:10.1109/iembs.1997.757084

[26] S. Aw et al. Head impulses reveal loss of individual semicircular canal function. Journal of Vestibular Research 9(3):173-180, 1999.

[27] J. Allum, L. Nijhuis, M. Carpenter. Differences in coding provided by proprioceptive and vestibular sensory signals may contribute to lateral instability in vestibular loss subjects. Exp Brain Res 184(3):391-410, 2007. http://dx.doi.org/10.1007/s00221-007-1112-z. DOI:10.1007/s00221-007-1112-z

[28] O. Findling et al. Trunk sway in mildly disabled multiple sclerosis patients with and without balance impairment. Exp Brain Res 213(4):363-370, 2011. http://dx.doi.org/10.1007/s00221-011-2795-8 DOI:10.1007/s00221-011-2795-8

[29] T. Kennie, G. Petrie. Engineering surveying technology 1990.
[30] F. Honegger, G. van Spijker, J. Allum. Coordination of the head with respect to the trunk and pelvis in the roll and pitch planes during quiet stance. Neuroscience 213:62-71, 2012. http:

//dx.doi.org/10.1016/j.neuroscience.2012.04.017 DOI:10.1016/j.neuroscience.2012.04.017

[31] M. Zadnikar, D. Rugelj. Postural stability after hippotherapy in an adolescent with cerebral palsy. Journal of Novel Physiotherapies 1(1), 2011. http://dx.doi.org/10.4172/2165-7025.1000106 DOI:10.4172/2165-7025.1000106

[32] C. Osler, R. Reynolds. Postural reorientation does not cause the locomotor after-effect following rotary locomotion. Exp Brain Res 220(3-4):231-237, 2012. http://dx.doi.org/10.1007/s00221-012-3132-6. DOI:10.1007/s00221-012-3132-6

[33] K. Altun, B. Barshan. Pedestrian dead reckoning employing simultaneous activity recognition cues. Meas Sci Technol 23(2):025103, 2012. http: //dx.doi.org/10.1088/0957-0233/23/2/025103 DOI:10.1088/0957-0233/23/2/025103.

[34] A. Gil-Agudo et al. A novel motion tracking system for evaluation of functional rehabilitation of the upper limbs. Neural regeneration research 8(19):1773, 2013.

[35] N. Ying, W. Kim. Use of dual euler angles to quantify the three-dimensional joint motion and its application to the ankle joint complex. Journal of Biomechanics 35(12):1647-1657, 2002. http: //dx.doi.org/10.1016/s0021-9290(02)00241-5 DOI:10.1016/s0021-9290(02)00241-5

[36] P. Brinckmann, W. Frobin, G. Leivseth. Musculoskeletal biomechanics. Thieme, 2002.

[37] I. Jolliffe. Principal component analysis. Wiley Online Library, 2002.

[38] S. Nash, A. Sofer. Linear and nonlinear programming. McGraw-Hill Inc., 1996.

[39] P. Wolf, C. Ghilani. Adjustment computations. Statistics and Least Squares in Surveying and GIS, John Wiley\&SSons 1997.

[40] W. Krzanowski. Principles of multivariate analysis: A userâĂŹs perspective. number 3 in oxford statistical science series, 1988.

[41] M. Hazewinkel. Encyclopaedia of mathematics, supplement III, vol. 13. Springer Science \& Business Media, 2001.

[42] M. Rocchi et al. The misuse of the confidence ellipse in evaluating statokinesigram. Ital J Sport Sci 12(2):169-172, 2005.

[43] J. Swanenburg et al. The reliability of postural balance measures in single and dual tasking in elderly fallers and non-fallers. BMC Musculoskeletal Disorders 9(1):162, 2008. http://dx.doi.org/10.1186/1471-2474-9-162, DOI:10.1186/1471-2474-9-162

[44] M. Moghadam et al. Reliability of center of pressure measures of postural stability in healthy older adults: Effects of postural task difficulty and cognitive load. Gait \& Posture 33(4):651-655, 2011. http: //dx.doi.org/10.1016/j.gaitpost.2011.02.016 DOI:10.1016/j.gaitpost.2011.02.016 
[45] H. Scheffe. The analysis of variance, vol. 72. John Wiley \& Sons, 1999.

[46] S. Baig et al. Cluster analysis of center-of-pressure measures. International Journal of Electrical and Computer Engineering 2012.

http://dx.doi.org/10.11159/ijecs.2012.002, DOI:10.11159/ijecs.2012.002.

[47] C. Greenwalt, M. Schultz. Principles of error theory and cartographic applications, acic technical report no. 961968.

[48] C. Jarque, A. Bera. A test for normality of observations and regression residuals. International Statistical Review / Revue Internationale de Statistique 55(2):163, 1987. http://dx.doi.org/10.2307/1403192 DOI:10.2307/1403192

[49] P. Kvam, B. Vidakovic. Nonparametric statistics with applications to science and engineering. John Wiley \& Sons, 2007.
[50] H. Diener et al. Quantification of postural sway in normals and patients with cerebellar diseases. Electroencephalography and Clinical Neurophysiology 57(2):134-142, 1984.

http://dx.doi.org/10.1016/0013-4694(84)90172-x DOI:10.1016/0013-4694(84)90172-x

[51] M. Patel et al. The effects of foam surface properties on standing body movement. Acta Oto-laryngologica 128(9):952-960, 2008.

http://dx.doi.org/10.1080/00016480701827517 DOI:10.1080/00016480701827517

[52] J. Blackburn et al. Kinematic analysis of the hip and trunk during bilateral stance on firm, foam, and multiaxial support surfaces. Clinical Biomechanics 18(7):655-661, 2003. http:

//dx.doi.org/10.1016/s0268-0033(03)00091-3 DOI:10.1016/s0268-0033(03)00091-3. 